\title{
STEINER POLYNOMIALS, WULFF FLOWS, AND SOME NEW ISOPERIMETRIC INEQUALITIES FOR CONVEX PLANE CURVES*
}

\author{
MARK GREEN ${ }^{\dagger}$ AND STANLEY OSHER ${ }^{\ddagger}$
}

0. Introduction. In this paper, we obtain some new inequalities for integrals of convex functions of the curvature (resp. Wulff curvature) of convex plane curves. We also show that the difference between the two sides of our inequalities are monotone decreasing as the region flows under the unit-speed outward normal (resp. Wulff) flow.

Given a bounded plane region $K$, the unit-speed outward normal flow has been highly studied, and is of interest in many applied problems, e.g. combustion. If instead $K$ grows by varying the outward normal speed to be a function $\gamma(\theta)$ of the direction of the unit normal, one has the Wulff flow, which is also of considerable interest, e.g. in studying the growth of crystals [O-M]. When the region $K$ is convex, there is a simple closed-form expression which describes these flows, and the region converges to a disk in the first case and a Wulff shape in the second. The area of the region when the initial region $K$ is convex is a polynomial in $t$, known respectively as the Steiner polynomial or Wulff-Steiner polynomial.

A novel feature of our approach is that we study the roots of the Steiner and WulffSteiner polynomials, which occur at negative values of $t$. The classical isoperimetric inequality in both cases states that these polynomials have (negative) real roots $t_{1} \geq t_{2}$, and that they are distinct if and only if $K$ is not a disc (respectively not a Wulff shape). Bonnesen's inequality states that the inradius and outradius $r_{i}$ and $r_{e}$ lie in the interval $\left[-t_{1},-t_{2}\right]$, and in the open interval if $K$ is not a disk (respectively not a Wulff shape). Our inequalities are most naturally stated and proved in terms of the roots $t_{1}$ and $t_{2}$. We feel that this is a potentially quite fruitful approach to studying convex bodies in higher dimensions.

In the context of this new approach, a very natural link between the outward normal and Wulff flows and the curvature integrals of the region appears. In important cases, the quantities that our inequalities state are positive are shown to be monotone decreasing as the region evolves under the flow. Particularly suggestive is the fact that the entropy of the curvature (respectively Wulff curvature) is bounded above in terms of the area and is monotone decreasing with time.

The inequalities themselves are quite fascinating. It came as a surprise to us that there are interesting new things to be said about convex plane curves. We state our inequalities here for arbitrary smooth bounded convex plane regions $K$ in the curvature case, and leave the Wulff case to the body of the paper. One of them is due to Gage [G], whose result was a source of inspiration to us. Gage's result is

$$
\int_{\partial K} k^{2} d s \geq \frac{\pi L}{A}
$$

which he points out fails for non-convex curves. For higher powers of the curvature

* Received February 9, 1999; accepted for publication March 10, 1999.

$\dagger$ Department of Mathematics, University of California, Los Angeles, CA 90095-1555, U.S.A. (mlg@math.ucla.edu). Research partially supported by NSF-DMS-9401256.

$\ddagger$ Department of Mathematics, University of California, Los Angeles, CA 90095-1555, U.S.A. (sjo@math.ucla.edu). Research partially supported by the NSF and DARPA through NSF-DMS9615854 and NSF-DMS-9706827. 
$m \geq 3$, the inequalities are new. For example,

$$
\int_{\partial K} k^{3} d s \geq \frac{L^{2} \pi-2 A \pi^{2}}{A^{2}}
$$

and

$$
\int_{\partial K} k^{4} d s \geq \frac{L^{3} \pi-3 A L \pi^{2}}{A^{3}}
$$

The general result is:

THEOREM 0.1. Let $K$ be a bounded convex plane region with radius of curvature $\rho(\theta)$ viewed as a function of the direction of the unit normal vector $\vec{n}(\theta)$, and let $t_{1} \geq t_{2}$ be the roots of the Steiner polynomial of $K$. Let $F(x)$ be a convex function on $\mathbf{R}^{+}$, i.e. $F^{\prime \prime}(x) \geq 0$. Then

$$
\frac{1}{2 \pi} \int_{S^{1}} F(\rho) d \theta \geq \frac{1}{2}\left(F\left(-t_{1}\right)+F\left(-t_{2}\right)\right),
$$

where $t_{1}, t_{2}$ are the roots of the Steiner polynomial of $K$.

Two other interesting cases of our inequalities, where $F$ is a transcendental function, the first of which can also be deduced from work: of Hamilton (see [GL]) and the second of which is new, are:

THEOREM 0.2. (Entropy inequality for curvature).

$$
\int_{\partial K} k \log (k \sqrt{A / \pi}) d s \geq 0 .
$$

Equality holds if $K$ is a disk.

THEOREM 0.3. Let

$$
e=\sqrt{1-\frac{4 \pi A}{L^{2}}}
$$

Then

$$
\int_{\partial K} \log (k \sqrt{A / \pi}) d s+\frac{L e}{2} \log \left(\frac{1+e}{1-e}\right) \leq 0 .
$$

Equality holds if $K$ is a disk.

We also obtain versions of these theorems for the Wulff case (see definition 1.13 below). For example, we have:

THEOREM 0.4. For $K, W$ bounded convex sets, $W$ symmetric, with support functions $p, \gamma$ respectively, and Wulff radius of curvature

$$
\rho_{W}=\frac{p+p^{\prime \prime}}{\gamma+\gamma^{\prime \prime}}
$$

we have for any convex function $F$,

$$
\frac{1}{2 A_{W}} \int_{S^{1}} F\left(\rho_{W}\right) \gamma\left(\gamma+\gamma^{\prime \prime}\right) d \theta \geq \frac{1}{2}\left(F\left(-t_{1}\right)+F\left(-t_{2}\right)\right)
$$

where $t_{1}, t_{2}$ are the roots of the Wulff-Steiner polynomial.

We also show: 
THEOREM 0.5. If in addition to the other hypotheses, $F^{\prime}(x)$ is concave, the difference between the two sides of the inequality in Theorems $0.1,0.2$, and 0.4 is monotone decreasing and for 0.3 is monotone increasing under the unit speed outward normal flow and Wulff flows respectively.

We note in particular the following physically intriguing result, which parallels work of Hamilton (see [GL]) for the inward curvature flow:

TheOREM 0.6. The entropy

$$
-\int_{\partial K} k \log (k \sqrt{A / \pi}) d s
$$

is increasing under the unit-speed outward normal flow.

An unusual feature of Theorem 0.1 is that its most natural statement involves the roots of the Steiner polynomial, which would appear to have no direct geometric significance, since they appear in the negative range of $t$-yet the roots can be eliminated from any given case and replaced by a function of $L$ and $A$. The proof of Theorem 0.1 involves three steps, each one a bit non-standard. The first step is an interesting "two-piece" version Jensen's inequality (Proposition 2.6). The second step is a bound for $-t_{2}$, i.e. for one of the roots of the Steiner polynomial, in terms of the largest integral $\int_{I} \rho(\theta) d \theta$ over a subset $I$ of $[0,2 \pi]$ of measure $\pi$ (Proposition 2.7). This inequality can be obtained for symmetric $K$ with some work from Bonnesen's inequality. The third step is a somewhat unusual symmetrization argument (Proposition 2.8). The end result of these new techniques is a new upper bound for the isoperimetric defect of a convex body in terms of the square of the $L^{1}$ norm of the difference of the radius of curvature and the average radius of curvature. The standard bound $[\mathrm{S}]$ is in terms of the square of the sup norm of the maximum and minimum of the radius of curvature.

Theorems $0.1-0.3$ and $0.5-0.6$ all generalize to the Wulff case. This is done in section 3. For example, one has:

THEOREM 0.7. For $W$ bounded convex symmetric with support function $\gamma$ and $K$ bounded convex with Wulff curvature $k_{W}$,

$$
\begin{aligned}
& \int_{\partial K} k_{W}^{2} \gamma d s \geq \frac{A_{W} L_{\gamma}}{A_{K}} \text { (Wulff Gage inequality); } \\
& \int_{\partial K} k_{W}^{3} \gamma d s \geq \frac{A_{W} L_{\gamma}^{2}-2 A_{W}^{2} A_{K}}{A_{K}^{2}} ; \\
& \int_{\partial K} k_{W} \log \left(k_{W} \sqrt{A_{K} / A_{W}}\right) \gamma d s \geq 0 \text { (Wulff entropy inequality). }
\end{aligned}
$$

In all three cases, the difference between the two sides of the inequality is monotone decreasing under the outward Wulff flow.

1. Convex plane curves, Steiner polynomials, and Wulff flows. In this section, we collect together some very classical facts about convex plane curves that exist in disparate sources (see [Sc] for an encylopaedic survey). We present them here from a novel perspective, in which the roots of the Steiner and Wulff-Steiner polynomials play a central role.

Definition 1.1. If $K \subseteq \mathbf{R}^{n}$ is a bounded convex region, then

$$
t K=\{t p \mid p \in K\}
$$


and if $K_{1}, K_{2} \subseteq \mathbf{R}^{n}$ are two bounded convex region, then

$$
K_{1}+K_{2}=\left\{p+q \mid p \in K_{1}, q \in K_{2}\right\} .
$$

We have the following well-known fact:

Proposition 1.2. For a bounded convex region $K$, the region inside the image of $\partial K$ under the constant speed outward flow at time $i \geq 0$ is $K+t B$, where $B$ denotes the unit ball.

DEFINITION 1.3. The support function $p$ of a bounded convex region $K$ is defined by

$$
p(\vec{v})=\max \{\vec{x} \cdot \vec{v} \mid \vec{x} \in K\}
$$

It is elementary that:

Proposition 1.4. If $K_{1}, K_{2}$ are bounded convex regions with support functions $p_{1}, p_{2}$, then the support function of $t_{1} K_{1}+t_{2} K_{2}$ is $t_{1} p_{1}+t_{2} p_{2}$.

Proposition 1.5. Let $W$ be a bounded convex region having support function $\gamma$. The image of a bounded convex region $K$ at time $t \geq 0$ under the normal flow having speed $\gamma(\vec{n})$ (the Wulff flow associated to $W$ ) is $K+t W$.

We now restrict ourselves to the case of bounded convex regions in the plane.

Proposition 1.6. Let $K$ be a bounded convex region in the plane having area $A$ and perimeter $L$, and where $\partial K$ has element of arc-length ds and curvature $k$. We have the following formulas:

(1) $d s=\left(p+p^{\prime \prime}\right) d \theta$;

(2) $k=1 /\left(p+p^{\prime \prime}\right)$;

(3) $A=(1 / 2) \int_{S^{1}} p\left(p+p^{\prime \prime}\right) d \theta$;

(4) $L=\int_{S^{1}}\left(p+p^{\prime \prime}\right) d \theta=\int_{S^{1}} p d \theta$;

(5) If $\vec{r}(\theta)$ is the position vector of the point of $\partial K$ whose normal vector is $\vec{n}(\theta)=$ $(\cos \theta, \sin \theta)$, then $\vec{r}(\theta)=p(\theta) \vec{n}(\theta)+p^{\prime}(\theta) \vec{n}^{\prime}(\theta)$.

Proof. We start from the basic fact

$$
p(\theta)=\vec{r}(\theta) \cdot \vec{n}(\theta) .
$$

Differentiating, we get

$$
p^{\prime}(\theta)=\vec{r}^{\prime}(\theta) \cdot \vec{n}(\theta)+\vec{r}(\theta) \cdot \vec{n}^{\prime}(\theta)=\vec{r}(\theta) \cdot \vec{n}^{\prime}(\theta) .
$$

It follows that

$$
\vec{r}(\theta)=p(\theta) \vec{n}(\theta)+p^{\prime}(\theta) \vec{n}^{\prime}(\theta),
$$

proving (5). Differentiating, we get

$$
\vec{r}^{\prime}(\theta)=p^{\prime}(\theta) \vec{n}(\theta)+p(\theta) \vec{n}^{\prime}(\theta)+p^{\prime \prime}(\theta) \vec{n}^{\prime}(\theta)+p^{\prime}(\theta) \vec{n}^{\prime \prime}(\theta) .
$$

Since

$$
\vec{n}^{\prime \prime}(\theta)=-\vec{n}(\theta)
$$

we have

$$
\vec{r}^{\prime}(\theta)=\left(p(\theta)+p^{\prime \prime}(\theta)\right) \vec{n}^{\prime}(\theta)
$$

Taking lengths, we get

$$
\frac{d s}{d \theta}=p+p^{\prime \prime}
$$


proving (1) and (2). The first part of (4) follows from (1), but then $\int_{S^{1}} p^{\prime \prime} d \theta=0$, giving the second formula. Now the element of area is given by

$$
\begin{aligned}
d A & =(1 / 2) \vec{r}(\theta) \wedge \vec{r}^{\prime}(\theta) d \theta \\
& =(1 / 2) p\left(p+p^{\prime \prime}\right) \vec{n} \wedge \vec{n}^{\prime} d \theta \\
& =(1 / 2) p\left(p+p^{\prime \prime}\right) d \theta,
\end{aligned}
$$

from which (3) follows.

CoRollary 1.7. Let $K$ be a bounded plane region with area $A$ and perimeter $L$. Let $A_{K}(t)$ denote the area of $K+t B$. Then for all $t \geq 0$,

$$
A_{K}(t)=A+L t+\pi t^{2} .
$$

Proof. By Proposition 1.4, the support function for $K+t B$ is $p+t$. It follows that

$$
\begin{aligned}
A_{K}(t) & =(1 / 2) \int_{S^{1}}(p+t)\left(p+t+p^{\prime \prime}\right) d \theta \\
& =(1 / 2) \int_{S^{1}} p\left(p+p^{\prime \prime}\right) d \theta+(1 / 2) t \int_{S^{1}}\left(2 p+p^{\prime \prime}\right) d \theta+(1 / 2) t^{2} \int_{S^{1}} d \theta \\
& =A+L t+\pi t^{2} .
\end{aligned}
$$

REMARK. This polynomial is called the Steiner polynomial of $K$.

A remarkable fact is that the behavior of the Steiner polynomial of $K$ for negative values of $t$ is quite interesting. For example, we have that

$$
\min \left(A_{K}(t)\right)=A\left(-\frac{L}{2 \pi}\right)=A-\frac{L^{2}}{4 \pi} .
$$

The isoperimetric inequality is thus equivalent to saying that

$$
\min \left(A_{K}(t)\right) \leq 0
$$

with equality if and only if $K$ is a disc.

DEFINITION 1.8. Let $t_{1} \geq t_{2}$ be the roots of $A_{K}(t)$. Let $r_{i}$ and $r_{e}$ be the radii of the largest inscribed and smallest circumscribed circles of $K$ respectively (called the inradius and outradius). Let $k$ be the curvature of $\partial K, \rho=\frac{1}{k}$ the radius of curvature, and $\rho_{\min }$ and $\rho_{\max }$ the minimum and maximum values of $\rho$. These quantities are all equal if $K$ is a disc.

REMARK 1.9. For the constant speed outward flow,

$$
A_{K+t B}(s)=A_{K}(t+s)
$$

and thus

$$
t_{i}(K+t B)=t_{i}(K)-t, \quad i=1,2
$$

If follows that

$$
\frac{d}{d t}\left(t_{i}(K+t B)\right)=-1 \quad i=1,2
$$


We have the formulae

$$
\begin{aligned}
& t_{1}=-\frac{L}{2 \pi}+\frac{\sqrt{L^{2}-4 \pi} \bar{A}}{2 \pi} \\
& t_{2}=-\frac{L}{2 \pi}-\frac{\sqrt{L^{2}-4 \pi} \bar{A}}{2 \pi} .
\end{aligned}
$$

The fact that the roots are real is equivalent to the isoperimetric inequality.

THEOREM 1.10. If $K$ is strictly convex and is not a disc, then

$$
-\rho_{\max }<t_{2}<-r_{e}<-\frac{L}{2 \pi}<-r_{i}<i_{1}<-\rho_{\min } \leq 0 .
$$

In particular,

$$
A\left(-\rho_{\min }\right)>0, \quad A\left(-\rho_{\max }\right)>0
$$

while if $-r_{e} \leq t \leq-r_{i}$,

$$
A(-t)<0
$$

REMARK. The last inequality more explicitly states that when $-r_{e} \leq t \leq-r_{i}$,

$$
A-L t+\pi t^{2}<0 \text {. }
$$

This is known as Bonnesen's inequality.

Proof. If we follow a given point inward under the constant speed normal flow, the time of first shock is $-\rho$. Thus up to $t=-\rho_{\min }$, the region remains convex and of positive area. Unless the region is a disk, the area remains positive even at $t=-\rho_{\min }$. This proves

$$
t_{1}<-\rho_{\min } .
$$

To prove Bonnesen's inequality, we first note the following lemma:

LEMMA 1.11. Let $C$ be either a maximal inscribed or minimal circumscribed circle for $K$. Then every closed semi-circle contains a point where $C$ is tangent to $\partial K$.

Proof of Lemma. We do the case of the inscribed circle, the other being similar. Let $v_{\alpha}, \alpha \in A$ be the unit vectors going from the center of $C$ to the points of tangency. If the conclusion fails, then there exists a unit vector $v$ such that $v \cdot v_{\alpha}<0$ for all $\alpha$-take $v$ pointing toward the midpoint of the semicircle that contains no $v_{\alpha}$. Now if we move the center of $C$ by $v \epsilon$ for $\epsilon>0$ small enough, then $C$ continues to lie in the interior of $K$ and has no points of tangency. So we may slightly increase the radius of $C$, contradicting maximality. This proves the lemma.

Returning to the proof of Bonnesen's theorem, choose points of tangency $\theta_{1}, \ldots$, $\theta_{N}$ on $C$ so that every half-circle contains one. Now

$$
A\left(-r_{i}\right)=\frac{1}{2} \int_{S^{1}}\left(p-r_{i}\right)\left(p+p^{\prime \prime}-r_{i}\right) d \theta .
$$

We now use the well-known:

LemMa 1.12. (Poincaré) Let $f$ be a function on $[0, a]$ whose first derivative is in $L^{2}$ and such that

$$
f(0)=f(a)=0 .
$$


Then

$$
\int_{[0, a]} f^{\prime}(x)^{2} d x \geq\left(\frac{\pi}{a}\right)^{2} \int_{[0, a]} f(x)^{2} d x
$$

with equality iff $f=A \cos (\pi x / a)+B \sin (\pi x / a)$. In particular, if $a \leq \pi$,

$$
\int_{[0, a]} f^{\prime}(x)^{2} d x \geq \int_{[0, a]} f(x)^{2} d x
$$

Equality holds iff

$$
f(x)=c \sin (\pi x / a)
$$

for some constant $c$.

Proof. By rescaling, we may take $a=\pi$. Extend $f$ to be an odd function on $[-\pi, \pi]$ and then extend to be periodic with period $2 \pi$. Now $f$ has first derivative in $L^{2}$, and if we expand by Fourier series

$$
f(x)=\sum_{n \in \mathbf{Z}} a_{n} e^{i n x}
$$

then $a_{0}=0$ since $f$ is odd. Now

$$
\int_{[0, \pi]}\left(f^{\prime}(x)^{2}-f(x)^{2}\right) d x=\pi \sum_{n \neq 0}\left(n^{2}-1\right)\left|a_{n}\right|^{2} .
$$

This gives the inequality since $n^{2} \geq 1$ for $n \neq 0$, and equality holds iff $a_{n}=0$ for $n \neq \pm 1$, and then from the boundary conditions $f(x)=c \sin (x)$.

Given this Lemma, by integration by parts we have

$$
A\left(-r_{i}\right)=\frac{1}{2} \int_{S^{1}}\left(\left(p-r_{i}\right)^{2}-\left(p^{\prime}\right)^{2}\right) d \theta .
$$

We can break up the right-hand side into integrals over the intervals $\left[\theta_{i}, \theta_{i+1}\right]$, and then apply the Lemma to see that $A\left(-r_{i}\right) \leq 0$. For equality to hold, we would need $p=r_{i}+a \cos \theta+b \sin \theta$, which gives a circle of radius $r_{i}$ with center $(-a,-b)$. The same argument works for $r_{e}$, and thus

$$
t_{2}<-r_{e}<-r_{i}<t_{1} .
$$

If we choose the origin to be the center of a maximal inscribed circle, then for all $\theta$, $r_{i} \leq p(\theta)$, integrating over the circle gives

$$
2 \pi r_{i} \leq \int_{S^{1}} p d \theta=L
$$

and it is easy to see we have equality only for a circle. A similar argument (with a different origin) gives

$$
L \leq 2 \pi r_{e} .
$$

So

$$
-r_{e}<-\frac{L}{2 \pi}<-r_{i}
$$

It remains to see that $-\rho_{\max }<t_{2}$. If we look at the curve whose support function is $p-t$ for $t \geq \rho_{\max }$, it is once again a convex curve (all points have gone past their shock, i.e. $\left.p-\rho_{\max } \leq 0\right)$, and its area is $A(-t)$, so we see that

$$
-\rho_{\max }<-t_{2} \text {. }
$$


This completes the proof.

All of this is equally valid in the Wulff case.

DeFINITION 1.13. Let $K, W$ be bounded convex regions in the plane having areas $A_{K}, A_{W}$ and having support functions $p, \gamma$ respectively. The Wulff length of $\partial K$ with respect to $W$ is

$$
L_{\gamma}=\int_{\partial K} \gamma d s
$$

The Wulff curvature is given by

$$
k_{W}=\frac{\gamma+\gamma^{\prime \prime}}{p+p^{\prime \prime}}
$$

and its inverse the Wulff radius of curvature is given by

$$
\rho_{W}=\frac{p+p^{\prime \prime}}{\gamma+\gamma^{\prime \prime}} .
$$

Let $A_{K, W}(t)$ denote the area of $K+t W$.

Proposition 1.14.

$$
A_{K, W}(t)=A_{K}+L_{\gamma} t+A_{W} t^{2}
$$

Proof. By Proposition 1.4, the support function of $K+t W$ is $p+t \gamma$. It follows that

$$
A_{K, W}(t)=(1 / 2) \int_{S^{1}}(p+t \gamma)\left(p+p^{\prime \prime}+t\left(\gamma+\gamma^{\prime \prime}\right)\right) d \theta
$$

and the proposition follows from expanding this in $t$.

DeFinition 1.15. Let $t_{1} \geq t_{2}$ be the roots of $A_{K, W}(t)$. Let

$$
r_{i}=\max \{t \mid \text { some translate of } t W \text { is contained in } K\}
$$

and

$$
r_{e}=\min \{t \mid \text { some translate of } t W \text { contains } K\}
$$

(called the $\mathrm{W}$-inradius and $\mathrm{W}$-outradius). Let $k_{W}$ be the Wulff curvature of $\partial K$, $\rho_{W}=\frac{1}{k_{W}}$ the Wulff radius of curvature, and $\rho_{\min }$ and $\rho_{\max }$ the minimum and maximum values of $\rho_{W}$. These quantities are all equal if $K=t W+\vec{a}$ for some tand $\vec{a}$.

THEOREM 1.16. For $K, W$ strictly convex, if $K \neq t W+\vec{a}$ for any $t$ and $\vec{a}$, then

$$
-\rho_{\max }<t_{2}<-r_{e}<-\frac{L_{\gamma}}{2 A_{W}}<-r_{i}<t_{1}<-\rho_{\min } \leq 0 .
$$

In particular,

$$
A_{K, W}\left(-\rho_{\min }\right)>0, \quad A_{K, W}\left(-\rho_{\max }\right)>0
$$

while if $-r_{e} \leq t \leq-r_{i}$,

$$
A_{K, W}(-t)<0
$$

The proof is essentially the same as the previous theorem.

There are a large number of classical results which are all consequences of this theorem, e.g. see pp. 119-121 of [S]. We note in particular: 
Corollary 1.17. (Wulff-Bonnesen inequality) If $K \neq s W+\vec{a}$ for any $s$ and $\vec{a}$ and $r_{i} \leq t \leq r_{e}$, then

$$
A_{K}-L_{\gamma} t+A_{W} t^{2}<0
$$

Proposition 1.18. For a convex bounded plane region $K$, the isoperimetric defect

$$
L^{2}-4 \pi A
$$

is an invariant of the outward unit-speed flow. For $K, W$ bounded convex plane regions, the Wulff isoperimetric defect

$$
L_{\gamma}^{2}-4 A_{W} A
$$

is an invariant of the outward Wulff flow.

Proof. This is an easy direct computation. However, the elegant way is to notice that these quantities can be computed from the minima of $A_{K}(t)$ and $A_{K, W}(t)$, and therefore are invariants of the flow.

Under the outward normal and Wulff flows, a region converges to a ball or Wulff shape. We will make crucial use of this fact in the second part of the paper.

Proposition 1.19. Let $K, W$ be bounded convex regions in the plane. Then as $t \rightarrow \infty$, the region $(1 / t)(K+t W)$ converges to $W$ in the strong sense that their support functions converge.

Proof. The support function of $(1 / t)(K+t W)$ is $(p / t)+\gamma$, and this converges to $\gamma$ as $t \rightarrow \infty$.

Proposition 1.20. Under the unit-speed outward normal flow, the curvature $k$ satisfies

$$
\frac{d \rho}{d t}=1 ; \quad \frac{d k}{d t}=-k^{2} .
$$

Under the Wulff flow for the region $W$ with support function $\gamma$, the Wulff curvature $k_{W}$ satisfies

$$
\frac{d \rho_{W}}{d t}=1 ; \quad \frac{d k_{W}}{d t}=-k_{W}^{2}
$$

Proof. The support function for $K+t W$ is $p+t \gamma$. Since $\rho_{W}=\left(p+p^{\prime \prime}\right) /\left(\gamma+\gamma^{\prime \prime}\right)$, we have that

$$
\begin{aligned}
\frac{d \rho_{W}}{d t} & =\frac{\left(\gamma+\gamma^{\prime \prime}\right)}{\gamma+\gamma^{\prime \prime}} \\
& =1
\end{aligned}
$$

Now

$$
\frac{d k_{W}}{d t}=\frac{d \rho_{W}^{-1}}{d t}=-k_{W}^{2}
$$

Taking $\gamma=1$ gives the result for the constant speed outward flow.

2. Some new inequalities of isoperimetric type for convex plane curves.

Throughout this section, let $K$ be a bounded convex plane region whose boundary has curvature $k$ and radius of curvature $\rho$. Let $t_{1}, t_{2}$ denote the roots of the Steiner polynomial of $K$. 
THEOREM 2.1. Let $F(x)$ be a convex function on $\mathbf{R}^{+}$, i.e. $F^{\prime \prime}(x) \geq 0$. Then

$$
\frac{1}{2 \pi} \int_{S^{1}} F(\rho) d \theta \geq \frac{1}{2}\left(F\left(-t_{1}\right)+F\left(-t_{2}\right)\right) \text {. }
$$

REMARK. We may rewrite this in the more geometrically appealing form

$$
\frac{1}{2 \pi} \int_{S^{1}} k F\left(\frac{1}{k}\right) d s=\frac{1}{2 \pi} \int_{S^{1}} \frac{F(\rho)}{\rho} d s \geq \frac{1}{2}\left(F\left(-t_{1}\right)+F\left(-t_{2}\right)\right) .
$$

For $F(x)=1 / x$, this is the Gage inequality [G]

$$
\int_{\partial K} k^{2} d s \geq \frac{\pi L}{A}
$$

which he points out fails for non-convex curves. For most other $F$ 's, the inequalities appear to be new. For example,

$$
\int_{\partial K} k^{3} d s \geq \frac{L^{2} \pi-2 A \pi^{2}}{A^{2}}
$$

and

$$
\int_{\partial K} k^{4} d s \geq \frac{L^{3} \pi-3 A L \pi^{2}}{A^{3}}
$$

Before coming to the proof, we state two further special cases:

THEOREM 2.2. (Entropy inequality for curvature)

$$
\int_{\partial K} k \log (k) d s+\pi \log \left(\frac{A}{\pi}\right) \geq 0
$$

Equality holds if $K$ is a disk.

REMARK. Note that either term in the inequality can be negative. An alternate formulation is

$$
\int_{\partial K} k \log (k \sqrt{A / \pi}) d s \geq 0
$$

THEOREM 2.3. Let

$$
e=\sqrt{1-\frac{4 \pi A}{L^{2}}}
$$

Then

$$
\int_{\partial K} \log (k \sqrt{A / \pi}) d s+\frac{L e}{2} \log \left(\frac{1+e}{1-e}\right) \leq 0 .
$$

Equality holds if $K$ is a disk.

REMARK. This may be stated more compactly as

$$
\int_{\partial K} \log \left(\left(\frac{1+e}{1-e}\right)^{e / 2} k \sqrt{A / \pi}\right) d s \leq 0 .
$$

We also have the following result: 
THEOREM 2.4. Let $F(x)$ be a $C^{3}$ convex function on $\mathbf{R}^{+}$such that $F^{\prime}(x)$ is concave, then the difference

$$
\frac{1}{2 \pi} \int_{S^{1}} F(\rho(\theta)) d \theta-\frac{1}{2}\left(F\left(-t_{1}\right)+F\left(-t_{2}\right)\right)
$$

is monotone decreasing under the unit-speed outward normal flow.

Proof. Under the unit-speed outward normal flow,

$$
\frac{d \rho}{d t}=1, \quad \frac{d t_{i}}{d t}=-1
$$

for $i=1,2$. The derivative of the difference in the theorem is

$$
\frac{1}{2 \pi} \int_{S^{1}} F^{\prime}(\rho(\theta)) d \theta-\frac{1}{2}\left(F^{\prime}\left(-t_{1}\right)+F^{\prime}\left(-t_{2}\right)\right) .
$$

Since $-F^{\prime}(x)$ satisfies the hypotheses of the Theorem 2.1, this quantity is $\leq 0$.

As a preliminary to proving the main theorem, we make the following definition and notation:

Definition 2.5. Consider

$$
\sup \left\{\int_{I} \rho(\theta) d \theta \mid I \subset S^{1}, \int_{I} d \theta=\pi\right\} .
$$

Let $I_{1}$ denote a subset of $S^{1}$ of measure $\pi$ realizing this bound, and let $I_{2}$ be its complement. There exists a real number a such that

$$
I_{1} \subseteq\{\theta \mid \rho(\theta) \geq a\}
$$

and

$$
I_{2} \subseteq\{\theta \mid \rho(\theta) \leq a\} .
$$

We set

$$
\rho_{1}=\frac{1}{\pi} \int_{I_{1}} \rho(\theta) d \theta
$$

and

$$
\rho_{2}=\frac{1}{\pi} \int_{I_{2}} \rho(\theta) d \theta .
$$

We note that

$$
\rho_{1}+\rho_{2}=\frac{L}{\pi} .
$$

Proposition 2.6. Let $F(x)$ be a convex function on $\mathbf{R}^{+}$. Then

$$
\frac{1}{2 \pi} \int_{S^{1}} F(\rho(\theta)) d \theta \geq \frac{1}{2}\left(F\left(\rho_{1}\right)+F\left(\rho_{2}\right)\right) .
$$

Proof. By Jensen's theorem, applied to $I_{i}$,

$$
\frac{1}{\pi} \int_{I_{i}} F(\rho(\theta)) d \theta \geq F\left(\frac{1}{\pi} \int_{I_{i}} \rho(\theta) d \theta\right)=F\left(\rho_{i}\right) .
$$

Taking the average of these two inequalities for $i=1,2$ gives the desired result.

Recall that a convex region is called symmetric if $p(\theta)=p(\theta+\pi)$ for all $\theta$. 
Proposition 2.7. For $K$ a symmetric bounded' convex region,

$$
\rho_{1} \geq-t_{2} \text {. }
$$

Proof. We first note that for a symmetric region $K$, if an inscribed circle of radius $r_{i}$ has center $C$, then a circle of radius $r_{i}$ and center $-C$ is also inscribed. By convexity, the circle with radius $r_{i}$ and centered at the origin is also inside $K$, and therefore

$$
p(\theta) \geq r_{i}
$$

for all $\theta$. By a similar argument,

$$
p(\theta) \leq r_{e}
$$

for all $\theta$. By the results of section 1 ,

$$
-t_{1} \leq p(\theta) \leq-t_{2}
$$

for all $\theta$.

We recall that

$$
-t_{1}=\frac{L}{2 \pi}-u, \quad-t_{2}=\frac{L}{2 \pi}+u,
$$

where

$$
u=\frac{\sqrt{L^{2}-4 \pi A}}{2 \pi} .
$$

Thus

$$
-u \leq p(\theta)-\frac{L}{2 \pi} \leq u
$$

for all $\theta$. Since $\rho_{1}+\rho_{2}=L / \pi$, we may write

$$
\rho_{1}=\frac{L}{2 \pi}+b, \quad \rho_{2}=\frac{L}{2 \pi}-b
$$

for some $b \geq 0$. The inequality we are trying to prove,

$$
\rho_{1} \geq-t_{2},
$$

is equivalent to proving

$$
b \geq u
$$

On $I_{1}$,

$$
\rho(\theta)-a \geq 0,
$$

so we have

$$
-\left(p(\theta)-\frac{L}{2 \pi}\right)(\rho(\theta)-a) \leq u(\rho(\theta)-a) .
$$

Integrating, we have

$$
-\frac{1}{\pi} \int_{I_{1}}\left(p(\theta)-\frac{L}{2 \pi}\right)(\rho(\theta)-a) d \theta \leq u\left(\rho_{1}-a\right) .
$$

On $I_{2}$,

$$
\rho(\theta)-a<0,
$$

so we have

$$
-\left(p(\theta)-\frac{L}{2 \pi}\right)(\rho(\theta)-a) \leq-u(\rho(\theta)-a) .
$$


Integrating, we have

$$
-\frac{1}{\pi} \int_{I_{2}}\left(p(\theta)-\frac{L}{2 \pi}\right)(\rho(\theta)-a) d \theta \leq-u\left(\rho_{2}-a\right) .
$$

Adding these two inequalities gives

$$
-\frac{1}{\pi} \int_{S^{1}}\left(p(\theta)-\frac{L}{2 \pi}\right)(\rho(\theta)-a) d \theta \leq u\left(\rho_{1}-\rho_{2}\right) .
$$

The left-hand side simplifies to

$$
\frac{2}{4 \pi^{2}}\left(L^{2}-4 \pi A\right)=2 u^{2} .
$$

The right-hand side is $2 u b$. The inequality is thus

$$
2 u^{2} \leq 2 u b
$$

and thus

$$
u \leq b
$$

as desired.

Proposition 2.8. For $K$ a bounded convex region (not necessarily symmetric),

$$
\rho_{1} \geq-t_{2}
$$

Proof. We proceed by a symmetrization argument. Given $K$, for any $\theta$ we divide $K$ into two regions by joining the points on $\partial K$ corresponding to $\theta, \theta+\pi$ by a straight line. Let $L_{1}, L_{2}$ be the lengths of the two pieces of $\partial K$ and $A_{1}, A_{2}$ the areas of the two halves; thus

$$
L=L_{1}+L_{2}, \quad A=A_{1}+A_{2} .
$$

Choose $\theta$ so that

$$
\left(2 L_{1}\right)^{2}-8 \pi A_{1}=\left(2 L_{2}\right)^{2}-8 \pi A_{2} ;
$$

this is possible because these quantities vary continuously with $\theta$ and are interchanged as $\theta$ runs from 0 to $\pi$, so by the intermediate value theorem their difference must go through 0 . Let $K_{1}$ be the symmetric convex body obtained by joining the first half of $K$ to a copy of itself rotated by 180 degrees, and $K_{2}$ a symmetric convex body obtained by doing the same thing to the second half. Thus $K_{i}$ has perimeter $2 L_{i}$ and area $2 A_{i}$ for $i=1,2$.

Now for symmetric convex regions, we may take the subset $I_{1}$ of the circle to be symmetric. Since $\int_{I} \rho(\theta) d \theta$ is maximized by $I_{1}$ among all subsets of measure $\pi$, it follows that

$$
\rho_{1}(K) \geq \frac{1}{2}\left(\int_{I_{1}\left(K_{1}\right)} \rho(\theta) d \theta+\int_{I_{1}\left(K_{2}\right)} \rho(\theta) d \theta\right)=\frac{1}{2}\left(\rho_{1}\left(K_{1}\right)+\rho_{1}\left(K_{2}\right)\right) .
$$

Now by Proposition 2.7 applied to the symmetric regions $K_{1}, K_{2}$,

$$
\rho_{1}\left(K_{1}\right) \geq \frac{2 L_{1}}{2 \pi}+\frac{\sqrt{\left(2 L_{1}\right)^{2}-8 \pi A_{1}}}{2 \pi}
$$

and

$$
\rho_{1}\left(K_{2}\right) \geq \frac{2 L_{2}}{2 \pi}+\frac{\sqrt{\left(2 L_{2}\right)^{2}-8 \pi A_{2}}}{2 \pi}
$$


Taking the average,

$$
\rho_{1}(K) \geq \frac{L_{1}+L_{2}}{2 \pi}+\frac{1}{2}\left(\frac{\sqrt{\left(2 L_{1}\right)^{2}-8 \pi A_{1}}}{2 \pi}+\frac{\sqrt{\left(2 L_{2}\right)^{2}-8 \pi A_{2}}}{2 \pi}\right) .
$$

We now use the way the decomposition was chosen to recall that

$$
\left(2 L_{1}\right)^{2}-8 \pi A_{1}=\left(2 L_{2}\right)^{2}-8 \pi A_{2} \text {. }
$$

Call this quantity $\beta$, so

$$
\rho_{1}(K) \geq \frac{L}{2 \pi}+\frac{\sqrt{\beta}}{2 \pi}
$$

The inequality we want is

$$
\rho_{1}(K) \geq \frac{L}{2 \pi}+\frac{\sqrt{\left(L_{1}+L_{2}\right)^{2}-4 \pi\left(A_{1}+A_{2}\right)}}{2 \pi} .
$$

We will be done if we can show that

$$
\beta \geq\left(L_{1}+L_{2}\right)^{2}-4 \pi\left(A_{1}+A_{2}\right) .
$$

Now

$$
\begin{aligned}
\beta & =2 L_{1}^{2}-4 \pi A_{1}+2 L_{2}^{2}-4 \pi A_{2} \\
& =\left(L_{1}+L_{2}\right)^{2}+\left(L_{1}-L_{2}\right)^{2}-4 \pi\left(A_{1}+A_{2}\right) \\
& \geq\left(L_{1}+L_{2}\right)^{2}-4 \pi\left(A_{1}+A_{2}\right) .
\end{aligned}
$$

This proves the proposition.

We have the elementary lemma:

LEMMA 2.9. If $F(x)$ is convex, then if $b \geq a \geq 0$ and $c$ arbitrary, then

$$
F(c-a)+F(c+a) \leq F(c-b)+F(c+b) .
$$

Proof. By the mean value theorem,

$$
F(c-a)-F(c-b)=(b-a) F^{\prime}\left(e_{1}\right)
$$

for some $c-b \leq e_{1} \leq c-a$ and

$$
F(c+b)-F(c+a)=\left(b-a_{i}\right) F^{\prime}\left(e_{2}\right)
$$

for some $c+a \leq e_{2} \leq c+b$. Thus $e_{2} \geq e_{1}$, and since $F^{\prime}(x)$ is increasing,

$$
F(c-a)-F(c-b) \leq F(c+b)-F(c+a),
$$

and the Lemma follows.

Proof of Theorem 2.1. We already have that

$$
\frac{1}{2 \pi} \int_{S^{1}} F(\rho(\theta)) d \theta \geq \frac{1}{2}\left(F\left(\rho_{1}\right)+F\left(\rho_{2}\right)\right) .
$$

Now

$$
\rho_{1}=\frac{L}{2 \pi}+b, \quad \rho_{2}=\frac{L}{2 \pi}-b
$$

and

$$
-t_{2}=\frac{L}{2 \pi}+u, \quad-t_{1}=\frac{L}{2 \pi}-u .
$$


By Proposition $2.8, b \geq u$, and so by Lemma 2.9 ,

$$
F\left(\rho_{1}\right)+F\left(\rho_{2}\right) \geq F\left(-t_{1}\right)+F\left(-t_{2}\right) .
$$

The Theorem follows by combining these two inequalities.

3. Isoperimetric inequalities in the Wulff case. Here the main result is:

TheOREM 3.1. For $K, W$ bounded convex sets, $W$ symmetric, with support functions $p, \gamma$ respectively, and Wulff radius of curvature $\rho_{W}$, we have for any convex $F(x)$,

$$
\frac{1}{2 A_{W}} \int_{S^{1}} F\left(\rho_{W}\right) \gamma\left(\gamma+\gamma^{\prime \prime}\right) d \theta \geq \frac{1}{2}\left(F\left(-t_{1}\right)+F\left(-t_{2}\right)\right)
$$

where $t_{1}, t_{2}$ are the roots of the Wulff-Steiner polynomial.

REMARK. One may re-write this as

$$
\frac{1}{2 A_{W}} \int_{S^{1}} k_{W} F\left(\frac{1}{k_{W}}\right) \gamma d s \geq \frac{1}{2}\left(F\left(-t_{1}\right)+F\left(-t_{2}\right)\right)
$$

where $k_{W}$ is the Wulff curvature.

We also have:

THEOREM 3.2. Let $F(x)$ be a $C^{3}$ convex function on $\mathbf{R}^{+}$such that $F^{\prime}(x)$ is concave. For $K$ convex and $W$ convex and symmetric, the difference

$$
\frac{1}{2 A_{W}} \int_{S^{1}} F\left(\rho_{W}\right) \gamma\left(\gamma+\gamma^{\prime \prime}\right) d \theta-\frac{1}{2}\left(F\left(-t_{1}\right)+F\left(-t_{2}\right)\right)
$$

is monotone decreasing under the outward Wulff flow.

Proof. The argument is the same as for Theorem 2.4, once we note that

$$
\frac{d \rho_{W}}{d t}=1
$$

and

$$
\frac{d t_{i}}{d t}=-1
$$

for $i=1,2$.

Proof of Theorem 3.1. The argument proceeds along very similar lines to that of section 2 , but with some modifications.

Definition 3.3. Consider

$$
\sup \left\{\int_{I} \rho_{W} \gamma\left(\gamma+\gamma^{\prime \prime}\right) d \theta \mid I \subset S^{1}, \int_{I} \gamma\left(\gamma+\gamma^{\prime \prime}\right) d \theta=A_{W}\right\}
$$

Let $I_{1}$ denote a subset of $S^{1}$ realizing this bound, and let $I_{2}$ be its complement. There exists a real number a such that

$$
I_{1} \subseteq\left\{\theta \mid \rho_{W}(\theta) \geq a\right\}
$$

and

$$
I_{2} \subseteq\left\{\theta \mid \rho_{W}(\theta) \leq a\right\}
$$

We set

$$
\rho_{1}=\frac{1}{A_{W}} \int_{I_{1}} \rho_{W} \gamma\left(\gamma+\gamma^{\prime \prime}\right) d \theta
$$


and

$$
\rho_{1}=\frac{1}{A_{W}} \int_{I_{2}} \rho_{W} \gamma\left(\gamma+\gamma^{\prime \prime}\right) d \theta
$$

We note that

$$
\rho_{1}+\rho_{2}=\frac{L_{\gamma}}{A_{W}}
$$

By Jensen's Theorem, we obtain by the same argument as in section 2 that:

Proposition 3.4. For $F(x)$ a convex function on $\mathbf{R}^{+}$, i.e. $F^{\prime \prime}(x) \geq 0$,

$$
\frac{1}{2 A_{W}} \int_{S^{1}} F\left(\rho_{W}\right) \gamma\left(\gamma+\gamma^{\prime \prime}\right) d \theta \geq \frac{1}{2}\left(F\left(\rho_{1}\right)+F\left(\rho_{2}\right)\right) \text {. }
$$

We can write

$$
\rho_{1}=\frac{L_{\gamma}}{2 A_{W}}+b, \quad \rho_{2}=\frac{L_{\gamma}}{2 A_{W}}-b
$$

and

where

$$
-t_{2}=\frac{L_{\gamma}}{2 A_{W}}+u, \quad-t_{1}=\frac{L_{\gamma}}{2 A_{W}}-u
$$

$$
u=\frac{\sqrt{L_{\gamma}^{2}-4 A_{K} A_{W}}}{2 A_{W}} .
$$

Proposition 3.5. If $K, W$ are symmetric, then

$$
\rho_{1} \geq-t_{2}
$$

i.e.

$$
b \geq u \text {. }
$$

Proof. By the argument of section 2, we may assume that the inscribed and circumscribed Wulff shapes are centered at the origin, and thus

$$
r_{i} \gamma(\theta) \leq p(\theta) \leq r_{e} \gamma(\theta)
$$

for all $\theta$, from which it follows that

$$
-u \gamma(\theta) \leq p(\theta)-\frac{L_{\gamma}}{2 A_{W}} \gamma \leq u \gamma(\theta)
$$

for all $\theta$. On $I_{1}$,

$$
\rho_{W}(\theta)-a \geq 0
$$

and thus

$$
-\left(p-\frac{L_{\gamma}}{2 A_{W}} \gamma\right)\left(\rho_{W}-a\right) \leq u \gamma\left(\rho_{W}-a\right)
$$

which integrates to

$$
-\frac{1}{A_{W}} \int_{I_{1}}\left(p-\frac{L_{\gamma}}{2 A_{W}} \gamma\right)\left(\rho_{W}-a\right)\left(\gamma+\gamma^{\prime \prime}\right) d \theta \leq u \rho_{1}-2 a
$$

On $I_{2}$, we obtain similarly

$$
-\frac{1}{A_{W}} \int_{I_{2}}\left(p-\frac{L_{\gamma}}{2 A_{W}} \gamma\right)\left(\rho_{W}-a\right)\left(\gamma+\gamma^{\prime \prime}\right) d \theta \leq-u \rho_{2}+2 a .
$$


Adding these, we get

$$
-\frac{1}{A_{W}} \int_{S^{1}}\left(p-\frac{L_{\gamma}}{2 A_{W}} \gamma\right)\left(\rho_{W}-a\right)\left(\gamma+\gamma^{\prime \prime}\right) d \theta \leq u\left(\rho_{1}-\rho_{2}\right) .
$$

The right-hand side is $2 u b$, and the left-hand side simplifies to

$$
\frac{L_{\gamma}^{2}-4 A_{K} A_{W}}{2 A_{W}^{2}}=2 u^{2}
$$

Thus

$$
2 u^{2} \leq 2 u b
$$

and hence $u \leq b$ as desired. This proves the proposition.

Proposition 3.6. For convex $K$ (symmetric or not) and $W$ symmetric, then

$$
\rho_{1} \geq-t_{2}
$$

i.e.

$$
b \geq u
$$

Proof. We symmetrize as in section 2, choosing points of $\partial K$ corresponding to $\theta, \theta+\pi$ with $\int\left(p+p^{\prime \prime}\right) \gamma d \theta$ equal to $L_{\gamma 1}, L_{\gamma 2}$ respectively and with each half having areas $A_{1}, A_{2}$ respectively. We choose $\theta$ so that $K_{1}, K_{2}$ with

$$
\left(2 L_{\gamma 1}\right)^{2}-8 A_{1} A_{W}=\left(2 L_{\gamma 2}\right)^{2}-8 A_{2} A_{W} .
$$

We get two symmetric convex regions $K_{1}, K_{2}$ with

$$
L_{\gamma}\left(K_{i}\right)=2 L_{\gamma i}
$$

and for $i=1,2$,

$$
A_{K_{i}}=2 A_{i}
$$

and

$$
L_{\gamma}(K)=L_{\gamma 1}+L_{\gamma 2}
$$

and

$$
A_{K}=A_{1}+A_{2} .
$$

As in section 2, it is easy to see that

$$
\rho_{1}(K) \geq \frac{1}{2}\left(\rho_{1}\left(K_{1}\right)+\rho_{2}\left(K_{2}\right)\right) .
$$

It follows from the symmetric case that

$$
\rho_{1}(K) \geq \frac{L_{\gamma}(K)}{2 A_{W}}+\sqrt{\beta}
$$

where

$$
\beta=\frac{\left(2 L_{\gamma 1}\right)^{2}-8 A_{1} A_{W}}{4 A_{W}^{2}}=\frac{\left(2 L_{\gamma^{2}}\right)^{2}-8 A_{2} A_{W}}{4 A_{W}^{2}} .
$$

We are thus reduced to showing that

$$
\beta \geq \frac{\left(L_{\gamma 1}+L_{\gamma 2}\right)^{2}-4 A_{W}\left(A_{1}+A_{2}\right)}{4 A_{W}^{2}} .
$$


This in turn is equivalent to showing that

$$
2 L_{\gamma 1}^{2}+2 L_{\gamma 2}^{2}-4 A_{W} A_{1}-4 A_{W} A_{2} \geq\left(L_{\gamma 1}+L_{\gamma 2}\right)^{2}-4 A_{W} A_{1}-4 A_{W} A_{2}
$$

which is equivalent to

$$
\left(L_{\gamma 1}-L_{\gamma 2}\right)^{2} \geq 0
$$

This completes the proof of the proposition.

\section{REFERENCES}

[G] M. E. GAGE, An isoperimetric inequality with applications to curve shortening, Duke Math J., 50 (1983), pp. 1225-1229.

[GL] M. E. GAGE AND Y. LI, Evolving plane curves by curvature in relative geometries II, Duke Math. J., 75:1 (1994), pp. 79-98.

[O-M] S. OSHER AND B. MERRIMAN, The Wulff shape as the asymptotic limit of a growing crystalline interface, Asian. J. of Math., 1 (1997), pp. 560-571.

[S] L. SAnTAlo, Integral Geometry and Geometric Probability, Encyclopedia of Mathematics and its Applications 1, Addison Wesley, Reading, Mass, 1976.

[Sc] R. SchneIder, Convex Bodies: the Brunn-Minkowski Theory, Cambridge University Press, Cambridge, England, 1993. 